Sammelbandes dar, da in allen Beiträgen darauf fokussiert wird, wie Wissen vermittelt wird. Diese Frage zieht sich wie ein roter Faden durch alle Beiträge. Außerdem sind die zusammengestellten Studien nachvollziehbar in kleine thematische Gruppen eingeteilt. Einige Analysen gewährleisten darüber hinaus einen Einblick in verschiedene Aspekte der Wissensvermittlung in anderen Sprachen als Deutsch. Beispielsweise werden auch französische oder englische Texte im Hinblick darauf untersucht. Dies bildet einen weiteren Vorteil der Publikation, weil man beobachten kann, ob sprachkontrastive Untersuchungen in diesem Bereich bestimmte aus der Perspektive der Medienlinguistik interessante Unterschiede entfalten können. Im Lichte des Gesagten kann die besprochene Veröffentlichung allen empfohlen werden, die sich für Medienlinguistik interessieren.

Marcelina Kałasznik

ORCID: 0000-0003-2713-5880

Uniwersytet Wrocławski, Polen

\title{
Maria José Dominguez Vásquez / Ulrich Engel / Gemma Paredes Suárez (2017): Neue Wege zur Verbvalenz I Theoretische und me- thodologische Grundlagen. Frankfurt am Main: Peter Lang Edition, 223 S. Reihe: Studien zur romanischen Sprachwissenschaft und in- terkulturellen Kommunikation. Herausgegeben von Gerd Wotjak. ISBN 978-3-631-71069-2.
}

DOI: $10.19195 / 0137-1169.37 .11$

Um sich in einer Fremdsprache effektiv verständigen zu können, ist die Beherrschung solcher Sprachkompetenzbereiche wie Aussprache, Morphologie, Syntax, Lexik und Pragmatik unentbehrlich. Lexikkenntnisse reichen nicht aus, um sprachlich korrekte und angemessene Äußerungen zu formulieren. Entscheidend sind hier die Regeln, mit denen einzelne Lexeme zu einer verständlichen Sprachstruktur verbunden werden. Vor allem Verben, die in der Mehrzahl der Fälle den Kern dieser Strukturen bilden, sind hier wichtig. Wie ein Verb andere Wörter konnotiert, so dass grammatisch korrekte Aussagen entstehen können, steht im Mittelpunkt der Valenztheorie. Valenzuntersuchungen spielen insbesondere in der Fremdsprachendidaktik eine große Rolle. Deswegen entstehen auf diesem Gebiet immer wieder neue Arbeiten, in denen Verbvalenzkontraste zweier oder mehrerer Sprachen dargestellt werden. Das hier rezensierte Werk „Neue Wege zur Verbvalenz I“ gehört ebenfalls dazu.

Die Zielsetzung der Autoren wird in der Einführung (Kapitel 1) präzise formuliert. Es geht ihnen einerseits darum, die Entwicklung der Dependenz- und Valenzgrammatik summarisch zu erfassen, andererseits neue Beschreibungsmethoden der Verbvalenz zu präsentieren (S. 9). Dazu charakterisieren sie die Verbvalenzkontraste zwischen deutschen und spanischen Verben und versuchen gleichzeitig, die vorhandene Lücke von Valenzuntersuchungen des Sprachenpaars Deutsch-Spanisch zu schließen. Die Wahl dieses Sprachenpaares begründen die Autoren damit, dass das Spanische zu den meistgesprochenen 
Sprachen der Welt gehört, dass auf dem Markt Valenzlexika des Spanischen fehlen, und zwar sowohl monolinguale als auch kontrastive deutsch-spanische, und dass es erhebliche Unterschiede zwischen beiden Sprachsystemen gibt (S. 10). Zur Zielgruppe des Buches rechnen die Autoren nicht nur Spanisch lernende Deutsche, Romanisten und Germanisten, sondern auch die, die sich allgemein für grammatische Probleme interessieren. Diese Gruppe würde ich zudem um diejenigen, die ein auf das Wesentliche beschränktes Kompendium der Valenz suchen, erweitern.

Der Einführung (S. 9-10) folgen sechs weitere Kapitel: Worum es geht (S. 11-14), Valenz (S. 15-38), Valenzlexikographie (S. 39-46), Einwände gegen die Valenztheorie (S. 47-60), Wie wir vorgehen wollen (S. 61-182) und Deutsch-Spanisches Wörterbuch: zur Auswahl des Verbbestands (S. 183-184). Die Arbeit schließt mit dem Inventar der grammatischen Begriffe (S. 185-194) und dem Literaturverzeichnis (S. 195-223). Es besteht hier keine eindeutige Unterteilung in einen theoretischen und einen praktischen Teil. Eventuell könnte man die ersten fünf Kapitel als „Grundlagen der Valenztheorie“ und das sechste Kapitel als „Beschreibungsmodell der Valenzkontraste von deutschen und spanischen Verben“ bezeichnen. Im Allgemeinen sehe ich das ganze Buch als eine umfassende theoretische Einführung zum darauffolgenden „Verbvalenzlexikon Deutsch-Spanisch“, mit dem es eine Ganzheit bildet.

Der Titel des zweiten dreiseitigen Kapitels - Worum es geht - sagt dem Leser eher nicht so viel. Nach der Lektüre würde ich den Titel am ehesten um die Worte in der Valenz erweitern. Der Leser bekommt hier vor allem allgemein erklärt, welche Rolle die Valenz in der Satzstruktur spielt. Von großer Bedeutung ist auch die Unterscheidung zwischen (Verb)Ergänzungen und Angaben und die Distinktion zwischen einem finiten und einem zentralen Verb. Kommt in einem Satz nur ein einziges Verb vor, decken sich die beiden Funktionen. In Sätzen mit mehrteiligem Verbalkomplex ist es aber das zentrale Verb, das alle Ergänzungen regiert. Die Unterscheidung wird mit Diagrammen illustriert (S. 12), was zusätzlich veranschaulichend ist. Das dritte Kapitel bietet dem Leser nicht nur eine genaue Definition der Valenz an, sondern auch eine diachronische Darstellung der Valenzforschungen, beginnend mit der Theorie von Lucien Tesnière (Unterkapitel 3.1). Im Jahre 1959 hat der französische Sprachwissenschaftler sein „Esquisse d'une syntaxe strukcturale“ publiziert, in dem der Begriff Valenz zum ersten Mal im linguistischen Sinne genutzt wurde. Ausführlich beschrieben wurde nicht nur die tesnierische Theorie, sondern auch ihre relevantesten Mängel. Ein bisschen merkwürdig ist für mich aber der Absatz, in dem diejenigen kritisiert werden, die das Buch Tesnières nur angelesen haben und es zu beurteilen wagen. Dass jedes Werk genau durchgelesen werden soll, um es begutachten zu können, ist eine Selbstverständlichkeit, die meines Erachtens keinen gesonderten Kommentar verlangt. Positiv zu bewerten ist aber die diachronische Fassung verschiedener valenzähnlicher Theorien (Unterkapitel 3.2). Unter deren Autoren befinden sich sowohl bekannte Namen wie Karl Bühler als auch Linguisten, die heute fast gar nicht mehr bekannt sind, wie Johann Werner Meiner (1781), August Grotenfend (1827) oder der Niederländer A. W. de Groot (1949). In Unterkapitel 3.3 dreht sich alles um den Begriff der Valenz. Der Leser bekommt einen umfassenden Überblick über die differenten Auffassungen des Valenzbegriffs. Neben der gewohnten Bezeichnung der Valenz als subklassenspezifische Rektion werden auch andere Herangehensweisen besprochen. Neben Argumentenpotential und Grundvalenz werden die Differenzen zwischen Valenzpotenz und Valenzrealisierung sowie zwischen Mikro- und Makrovalenz eingehend erklärt. Wichtig 
zu erwähnen ist in diesem Zusammenhang zudem, dass die Autoren ihr Augenmerk auf die Substantiv- und Adjektivvalenz legen. Den beiden Phänomenen könnte aber vielleicht ein bisschen mehr Platz gewidmet werden. Was den Valenzträger betrifft, so wird noch auf drei Tendenzen hingewiesen und zwar darauf, dass einige Linguisten nur Verben Valenz zuschreiben, während für andere auch andere Wortarten Valenz haben können. Die dritte Gruppe von Linguisten schließlich fasst die Valenz am weitesten, sie geht neben der Verb-, Substantiv- oder Adjektivvalenz zusätzlich von einer phonologischen oder morphologischen Valenz aus. Eine für die Valenztheorie epochale Veröffentlichung war ohne Zweifel der Aufsatz von Charles Fillmore „The Case for Case“. Mit der Einführung von „deep cases" haben die Grammatiker auf die semantische Seite der Valenz aufmerksam gemacht. In „Neue Wege ...“ haben die Autoren die Theorie Fillmores aufgegriffen, was zugleich eine Einleitung zur Erklärung der semantischen Valenz darstellt. Interessant und informativ ist hier die synthetische Darstellung der Entwicklung der Valenztheorie und deren Einfluss einerseits auf die Grammatik, was die Entstehung neuer Grammatiktypen wie der Valenzgrammatik oder der Konstruktionsgrammatik zur Folge hat, anderseits auf die Lehrwerke und Grammatiken für DaF.

In der Valenzlexikographie (Kapitel 4) werden nicht nur deutsche Valenzlexika vorgestellt, sondern auch bilinguale und kontrastive (auch geplante) Valenzwörterbücher mit Deutsch als Bezugssprache angeführt. Darüber hinaus findet man Informationen zu laufenden, nicht nur deutschen, Valenz-Projekten, wie z.B. The Contagram Dutch-FrenchEnglish Contrastive Verb Valency oder zum dänischen Odense Valency Dictionary. Auch Monographien, Wörterbücher und Lexika zur Substantiv- und Adjektivvalenz, obwohl beide Valenztypen nicht so intensiv beforscht wurden wie die Verbvalenz, werden hier angesprochen. Solch eine umfassende Zusammenstellung der Valenz-Werke ist zweifellos lobenswert. Man hat den Eindruck, dass die Autoren kein einziges Valenzlexikon oder - wörterbuch des Deutschen vergessen haben. Ein ähnlich umfassender und informativer Überblick über die Dependenz- und Valenztheorie im Spanischen bekommt man in Unterkapitel 3.2. In Unterpunkten erhält der Leser Informationen über Grammatiken mit Valenzeinflüssen, deutsch-spanische Grammatiken, mono- und bilinguale Valenzwörterbücher und lexikographische kontrastive Projekte zum Spanischen und Deutschen.

In der Geschichte der Valenzforschungen ist an dieser Theorie, begründet oder unbegründet, viel Kritik geübt worden. Ungezählte Linguisten haben in ihren Veröffentlichungen auf unzählige Zweifelsfälle hingewiesen. ${ }^{1}$ Genau mit diesen Fällen setzen sich die Autoren im fünften Kapitel auseinander. Sie gehen auf die wichtigsten Vorbehalte und Einwände zum Thema Valenz ein. Der erste und wahrscheinlich auch der meistdiskutierte Fall betrifft die Abgrenzung der Ergänzungen von Angaben. Es werden hier unterschiedliche Testverfahren angeführt, die aus der IDS-Grammatik stammen: Reduktions-, Implikations- und Anschlusstest, sowie die Vorschläge von Gerd Wotjak, der zwischen variablen/konstanten Argumenten und variablen/konstanten Modifikatoren unterscheidet. Die weiteren Probleme beziehen sich auf Valenzerweiterungen und Valenzreduktionen, die verblose Beschreibung von Direktivergänzungen, und auf die Unfähigkeit der Valenz, die Wortstellung, die Finitendungen der Verben und die Textkonnexion zu erklären. Die Schlüssigkeit der Argumentation der Autoren steht dabei außer Frage.

${ }^{1}$ Das Buch Kontra Valenz von Joachim Jacobs darf nicht unerwähnt bleiben. 
Während in den Kapiteln 1-5 auf die Valenztheorie eingegangen wird, geht das sechste Kapitel umfassend darauf ein, was im deutsch-spanischen Valenzlexikon zu erwarten ist. Die Ausgangssprache ist Deutsch, wobei die Liste der beschriebenen Verben 121 Einheiten umfasst und auf der bewährten Liste von Zertifikat Deutsch basiert. Das Beschreibungsmodell der Verben enthält die Stammformen, die Bedeutung, die syntaktische und die semantische Valenz, sowie die Ausbaumöglichkeiten der Ergänzungen zu satzartigen Formen. Außerdem bildet die Metataxe eine separate Rubrik (Unterkapitel 6.9), in der die unterschiedlichen Äquivalente der Ergänzungsklassen exponiert werden. Im Übrigen fehlt es auch nicht an Informationen zu Passivfähigkeit und Passivbildung (Unterkapitel 6.12). Die Autoren beschreiben die möglichen Passivformen, wobei sie einen besonderen Schwerpunkt auf das Spanische legen, weil es in diesem Bereich bedeutende Differenzen gibt und ,häufig [...] keine passivische Entsprechung zum deutschen Passiv vorliegt" (S. 168). Es werden auch für Valenzlexika eher untypische Daten berücksichtigt, wie mögliche Wortbildungsformen (Unterkapitel 6.6.) insbesondere bei Verben, Nomina und Adjektiven. Wenn man darüber hinaus noch berücksichtigt, dass bei jedem Verb die Beschreibung jeweils mit Beispielen und eventuellen zusätzlichen Anmerkungen versehen wird, gehört das hier verwendete Beschreibungsmodell ohne Zweifel zu den umfangreichsten, die man in kontrastiven Valenzlexika finden kann.

Das Buch „Neue Wege zur Verbvalenz I” ist ein sehr gutes und empfehlenswertes Werk, nicht nur weil es eine Lücke in der deutsch-spanischen kontrastiven Valenzforschung schließt, sondern auch, weil es eine hervorragende Quelle von allgemeinem Wissen über die Valenz ist. In einem etwas mehr als 200-seitigen Buch findet der Leser sowohl eine ausführliche Definition der Valenz, als auch wertvolle Informationen u.a. zur Geschichte der Valenzforschung, zu Valenzlexika und -wörterbüchern sowie zur syntaktischen und semantischen Valenz. Die Autoren haben zudem ein Inventar der grammatischen Begriffe erstellt. Jeder, der sich mit der Valenztheorie beschäftigt oder beschäftigen will, sollte zum vorliegenden Werk greifen, selbst wenn er keine Spanischkenntnisse hat.

Michat Smułczyński

ORCID: 0000-0003-3699-4724

Uniwersytet Wrocławski, Polen 